\title{
TTR
}

Traduction, terminologie, re?daction

\section{Quelques problèmes de traduction de textes de théâtre latin}

\section{André Daviault}

Volume 3, numéro 1, 1er semestre 1990

L'agora de la traduction

URI : https://id.erudit.org/iderudit/037058ar

DOI : https://doi.org/10.7202/037058ar

Aller au sommaire du numéro

Éditeur(s)

Association canadienne de traductologie

ISSN

0835-8443 (imprimé)

1708-2188 (numérique)

Découvrir la revue

Citer cet article

Daviault, A. (1990). Quelques problèmes de traduction de textes de théâtre latin. TTR, 3(1), 49-61. https://doi.org/10.7202/037058ar d'utilisation que vous pouvez consulter en ligne.

https://apropos.erudit.org/fr/usagers/politique-dutilisation/ 


\title{
Quelques problèmes de traduction de textes de théâtre latin
}

\author{
André Daviault
}

Ce bref essai porte sur les problèmes ainsi que sur la méthodologie propres à la traduction de textes latins, et plus spécialement du théâtre comique, et présente quatre cas pratiques représentatifs tout à fait accessibles à des non-latinistes.

La littérature latine antique est constituée par un ensemble d'œuvres qui s'étendent sur une période d'environ sept siècles (du $\mathrm{III}^{e} \mathrm{~s}$. avant J.-C. au IVe s. après J.-C.). Outre le fait que ce corpus n'est pas encore entièrement traduit, les innombrables traductions qui existent sont régulièrement reprises dans le but de procurer une compréhension plus sûte de ces textes.

Nous sommes un peu par rapport au latin dans la même situation que l'historien grec Polybe qui devait opérer pour le bénéfice de ses contemporains et compatriotes du $\mathrm{II}^{e} \mathrm{~s}$. avant $\mathrm{J}$.-C. la transposition dans sa propre langue des réalités romaines. Mais notre éloignement temporel de cette civilisation mixte, espèce de synthèse d'acculturations successives, élève la quantité de la difficulté à une puissance exponentielle. N'oublions pas que notre vision de cet univers antique est celle d'étrangers d'un autre âge. Sans insister outre mesure sur la nature de l'effort qu'il faut consentir pour se rapprocher de la langue latine, il convient d'être conscient que notre regard à distance risque d'être déformant et qu'une grande prudence ainsi qu'une expérience sans cesse perfectible des choses romaines doivent guider nos interprétations.

On n'aura pas de peine à comprendre que, si l'entreprise de traduction de ce qu'il est convenu d'appeler une langue morte en 
une langue moderne est loin d'être désespérée, au contraire, il n'en reste pas moins des passages dont l'appréhension n'est pas sans rapport avec le mécanisme de la conjecture en critique textuelle. Il serait vain de dissimuler le rôle de l'appréciation personnelle dans la résolution de certains cas et donc la part de subjectivité et d'incertitude d'un tel exercice. Mais avec cette espèce d'avertissement nous n'avons pas l'outrecuidance de prétendre que la partie traduite du corpus latin n'est qu'un équivalent approximatif des cuvres originales; toutefois il convient d'attirer l'attention sur le caractère particulier de cette entreprise de décodage du message latin qui se poursuit et se perfectionne depuis la Renaissance.

En guise de prologue, et même s'il n'appartient pas au répertoire du théâtre, nous ferons état d'un exemple qui est typique de ces aggiornamenti. Il se rapporte à l'auteur du Satiricon et consiste en un témoignage de l'historien Tacite, d'une cinquantaine d'années postérieur à Pétrone. Tacite, faisant la relation du suicide de Pétrone, raconte que celui-ci sur le point de mourir «traça sous les noms d'hommes et de femmes corrompus le récit des débauches du Prince dans leurs plus monstrueuses recherches et lui envoya cet écrit cacheté»' (flagitia principis sub nominibus exoletorum feminarumque et nouitatem cuiusque stupri perscripsit). On se souvient du problème irritant que pose ce passage: comment le récit de Tacite pourrait-il porter sur notre Satiricon, qui n'est pas précisément un recueil de perversités et dont nous n'avons conservé que quelques chapitres, mais qui ont déjà la taille d'un livre de poche? Comment Pétrone aurait-il pu, la veille de sa mort, écrire en une journée un texte dont la rédaction a dû prendre, il est facile de le présumer, au moins quelques années? Le réflexe traditionnel a été de croire que l'historien parlait d'autre chose, d'un second écrit, beaucoup plus court, de Pétrone.

Dans le cadre d'une récente recherche (1988), nous nous sommes intéressé à cette question de savoir si le Satiricon et l'extrait de Tacite étaient réconciliables ${ }^{2}$. En réalité il fallait moins se demander si le dernier envoi de Pétrone était ou non le Satiricon que d'essayer de voir s'il présentait un lien avec lui. Car il paraissait difficile d'adhérer sans réserve à la fable d'un Pétrone, qui,

1. Tacite, Annales, XVI, 17, 18 et 19. Traduction d'Alfred Emout, Collection Guillaume Budé des Universités de France (Paris, Les Belles Lettres, 1923).

2. André Daviault, "Satiricon de Pétrone ou les mille et une nuits d'un agent double", le Risque de lire (Département des littératures de l'Université Laval, Nuit blanche èditeur, 1988), pp. 139-161. Voir aussi Carlo Corbato, "Tacito, Ann. XVI,19: considerazioni sulla tradizione del Satyricon di Petronion, Miscellanea di studi classici in onore di Eugenio Manni (Rome, Georgio Bretschneider, 1980), vol. II, pp. 565-572. 
dans les derniers moments de sa vie, aurait fabriqué contre Néron une espèce de pamphlet violemment ironique sans aucune parenté avec son grand-œuvre satirique. Tout le problème repose sur le sens qu'il convient de reconnaître au verbe PERSCRIPSIT qui vient d'être traduit par «il traça le récit». On a automatiquement pris le terme dans son acception la plus courante de diligenter scribere, "écrire en détail», sans s'aviser de la signification technique bien attestée de l'expression sub nominibus perscribere, «entegistrer avec les noms», «mettre les noms dans un registre» (Corbato, 1980, pp. 561-572).

Quand on se représente ce qu'était le Satiricon, c'est-à-dire une satire clandestine, qui ridiculisait sous le masque de la fiction tout ce qui concernait Néron et son entourage, on subodore facilement la portée qu'est susceptible d'avoir l'observation de Tacite: l'historien pourrait laisser entendre que Pétrone, avant l'issue fatale qu'il s'était assignée, avait décidé de confirmer au principal intéressé le sens de son énigmatique entreprise littéraire: Tacite nous indique, à notre avis, que Pétrone avait résolu de donner les clés de son récit apparemment fictif en notant cette fois avec les noms, entendons les noms authentiques, les aventures intimes dont certains personnages et épisodes romanesques cachaient le souvenir. On conviendra que, si cette vision des choses est la bonne, le peu de temps imparti à «l'arbitre des élégances» lui suffisait cependant pour procurer ces explications, qui éclairaient l'œuvre, mais ne la constituaient pas. Pour nous le dernier écrit de Pétrone a bien des chances d'être une émanation du Satiricon, la signature du Satiricon. Telle est l'information transmise par Tacite que je traduirais par quelque chose comme uen donnant le nom de ses complices, hommes et femmes corrompus, il authentifia les débauches du Prince et leurs plus monstrueuses recherches».

Venons-en maintenant aux problèmes de traduction relatifs aux textes de théâtre latin que je souhaiterais soumettre à votre attention: il s'agit d'un petit corpus particulier, qui ne doit guère avoir d'équivalent dans la littérature moderne, et qui est constitué par une collection de citations sans lien les unes avec les autres et tirées de diverses comédies d'auteurs différents des $\mathrm{II}^{\mathrm{e}}$ et $\mathrm{I}^{\mathrm{er}}$ siècles avant J.-C. Ces citations nous ont été transmises par des sources indirectes, c'est-à-dire par des lexicologues ou des grammairiens de basse époque qui les reproduisaient à titre d'exemples pour illustrer l'emploi d'une tournure ou d'une expression archaïques. C'est un peu comme si nous n'avions plus aujourd'hui de Shakespeare ou de Molière que quelques centaines de vers sélectionnés çà et là dans différentes œuvres disparues.

Lintérêt de l'examen de ces fragments est évident pour le linguiste et l'historien de la langue latine, mais il existe également 
pour le littéraire et le spécialiste du théâtre. Il y a donc du travail pour leur traducteur, qui contribuera à améliorer notre connaissance du génie comique des Romains, également caractéristique de leur mentalité. Paradoxalement nous allons examiner ensemble les écueils susceptibles de gêner ou même de compromettre l'acte de traduction. Aux difficultés évoquées plus haut inhérentes à l'étude de tout texte latin, s'ajoute celle considérable de l'absence de tout contexte. Naturellement le but n'est pas de chercher à renconstituer des scénarios, mais plus modestement de tâcher de percer le sens d'une phrase.

Si on y arrive la plupart du temps, il reste plusieurs cas de résistance. Il est entendu que le commentaire du grammairien citateur peut éclairer le passage en question: ainsi il faut rendre justice au lexicologue compilateur Festus sans qui ces deux extraits de deux poètes comiques seraient restés sibyllins.

Voici le premier:

$\mathrm{Si}$ on applique un tentipellium, les rides se détendent sur le visage $^{3}$.

et le second:

À la place des mains, moi, je crois qu'ils ont un tentipellium ${ }^{4}$.

En dépit de l'étymologie transparente du mot tentipellium, formé de tendere, «tendre», et de pellis, «peau», la distinction de Festus demeure indispensable à la compréhension des vers: il nous informe que dans le premier exemple le terme désigne un produit cosmétique, une pommade, une espèce de masque facial ${ }^{5}$, et que dans le second il évoque un appareil, une forme de cordonnier, qui lui sert à étirer les peaux ${ }^{6}$. La connaissance des textes comiques grecs et latins fait le reste et nous aide à identifier les cibles de ces deux remarques qui visent, l'une, le facies trop ridé d'un personnage d'âge avancé, l'autre, les mains voleuses des esclaves de la Comédie, probablement des cuisiniers, que Plaute compare souvent aux griffes ou crocs des milans ${ }^{7}$.

3. Titinius, 168-169 Daviault (=173-174 Ribbeck): Tentipellium si inducitur,/rugae in ore extenduntur.

4. Afranius, 284 Daviault (=281 Ribbeck): Pro manibus credo habere ego illos tentipellium.

5. Festus, De uerborum significatu, 500 Lindsay: Titinium autem Verrius existimare id medicamentum esse.

6. Festus, ibid.: Tentipellium Artorius putat esse calciamentum ferratum, quo pelles extenduntur indeque Afranium dixisse in Promo.

7. Plaute, Casina, 720; Pseudolus, 852 et 887. 
Nous n'avons pas toujours la bonne fortune de posséder ces précisions. Jetons un coup d'œil sur un autre fragment dont les termes latins n'offrent aucun secret, mais qui une fois lu ou traduit n'est pas très évident; il s'agit de la réplique d'un acteur rapportant la réflexion d'un tiers et qui dit:

\section{Il affirme que l'ours l'a mordu'.}

Linterprétation des fragments ne se limite pas à une approche purement philologique. On peut fort bien comme ici comprendre le sens d'une phrase sans en saisir la portée. Le traducteur pourrait prendre la réflexion au pied de la lettre et considérer qu'elle provient d'un récit, concluant que l'auteur a eu recours au procédé dramatique ordinaire pour raconter un épisode qu'il n'était pas indispensable de montrer sur scène: il interpréterait le fragment comme l'allusion d'un personnage à un danger auquel un autre a échappé. L'hypothèse d'un récit comique ou d'un conte à dormir debout épuise-t-elle le champ des possibilités? On pourrait encore penser que le poète, dans une mise en scène aussi réaliste que spectaculaire, a porté sur les tréteaux un ours véritable ou plus probablement un acteur revêtu d'une peau d'ours comme la bête de la comédie The Winter's Tale de Shakespeare. De fait nul n'ignore la prédilection des Romains pour les mises en scène extraordinaires, cependant à l'époque à laquelle appartient ce fragment, c'est-à-dire au début du I ${ }^{\text {er }} \mathbf{s}$. avant J.-C., les exagérations de la mise en scène n'avaient pas encore commencé de déformer les représentations théâtrales, ce qui allait se produire une génération plus tard.

Pourtant cette mention ou plutôt cette intervention d'un ours dans le scénario d'une ancienne comédie latine a quelque chose de singulier et en essayant de l'expliquer, sinon par l'apparition surprenante de la bête sur la scène, du moins par le récit d'un tel épisode, nous ne vidons pas la question. Il faut chercher ailleurs la signification de ce passage. Il est indispensable de tirer parti de toutes les sources de connaissance, qu'elles soient linguistiques, historiques, littéraires ou encore épigraphiques. Précisément un rapprochement avec des documents épigraphiques nous procurera la clé du problème. Portons attention à deux graffiti pompéiens et à un troisième découvert sur le Palatin, qui témoignent tous d'un tour proverbial où il est question de l'ours:

8. Atta, 7 Daviault ( $=6$ Ribbeck): Vrsum se memordisse autumat. 
Que les ours me dévorent ainsi que moi le couillon qui lis cette inscription!?

Tu vas grossir. Le rival qui fera l'amour avec ma maîtresse, que dans le recoin de la montagne l'ours le mange! ${ }^{10}$

Que les ours me dévorent! ${ }^{11}$

Ces vestiges reproduisent une formule purement latine, une locution porteuse de malédiction qui doit être placée sur le même pied que notre expression «que le diable l'emporte». Comme Jean-Pierre Cèbe et Paul Veyne (1965) l'ont démontré, il arrivait aux Romains de modifier ce type de formules et de s'en amuser: ainsi Pétrone fait dire à un invité de Trimalchion qui veut justifier spirituellement la voracité avec laquelle il avale la viande d'ours au menu, que si l'ours mange le chétif humain, à plus forte raison le chétif humain doit manger de l'ours, laissant entendre par là que si (sous-entendu: selon la maxime) l'ours mange l'homme, alors celui-ci doit lui rendre la pareille ${ }^{12}$. D'une manière tout à fait comparable, le poète comique de notre fragment a simplement dénaturé cet ancien dicton populaire et l'a mis dans la bouche de quelqu'un qui indique d'une façon bouffonne qu'il a eu une malchance, comme il dirait qu'il a reçu une tuile sur la tête.

Mais laissons là les fragments et occupons-nous d'une cuvre intégrale que nous avons tout entière sous les yeux (voir illustrations): il s'agit d'inscriptions latines du III $^{e} s$. ou du IV ${ }^{e} s$. après J.-C. accompagnant à la manière de bulles de bandes dessinées une scène figurće sur mosaïque qui vient d'être découverte à Puente Genil en Andalousie dans la province de Cordoue: elles font partie de la décoration du pavement d'une pièce comportant quatre absides autour d'un carré et qui était une salle de réception ou une salle de bains de la villa d'un riche latifundiaire hispano-romain. L'artiste a exploité un sujet unitaire, c'est-à-dire le thème nilotique de la géranomachie ou la lutte légendaire des grues contre les pygmées, déjà attestée chez Homère. Le spectateur était donc invité à suivre une scène narrative en quatre épisodes dans laquelle il y avait exceptionnellement association d'un texte suivi, un dialogue, avec chaque personnage (et non pas une simple mention du nom des personnages comme c'est habituellement le cas): les

9. Corpus Inscriptionum Latinarum (=C.I.L.), 4, 2360: Vrsi me comedant et ego uerpam qui lego!

10. C.I.L., 4, 1645: Cresces. Quisquam meam futuet riualis amicam illum secretis montibus ursus edat!

11. C.I.L., 4, 4951: Vrsi me comedant!

12. Jean-Pierre Cèbe et Paul Veyne, «Proverbes chez Pétrone», Annales de la Faculté des Lettres d'Aix-en-Provence (1965), pp. 173-176. 

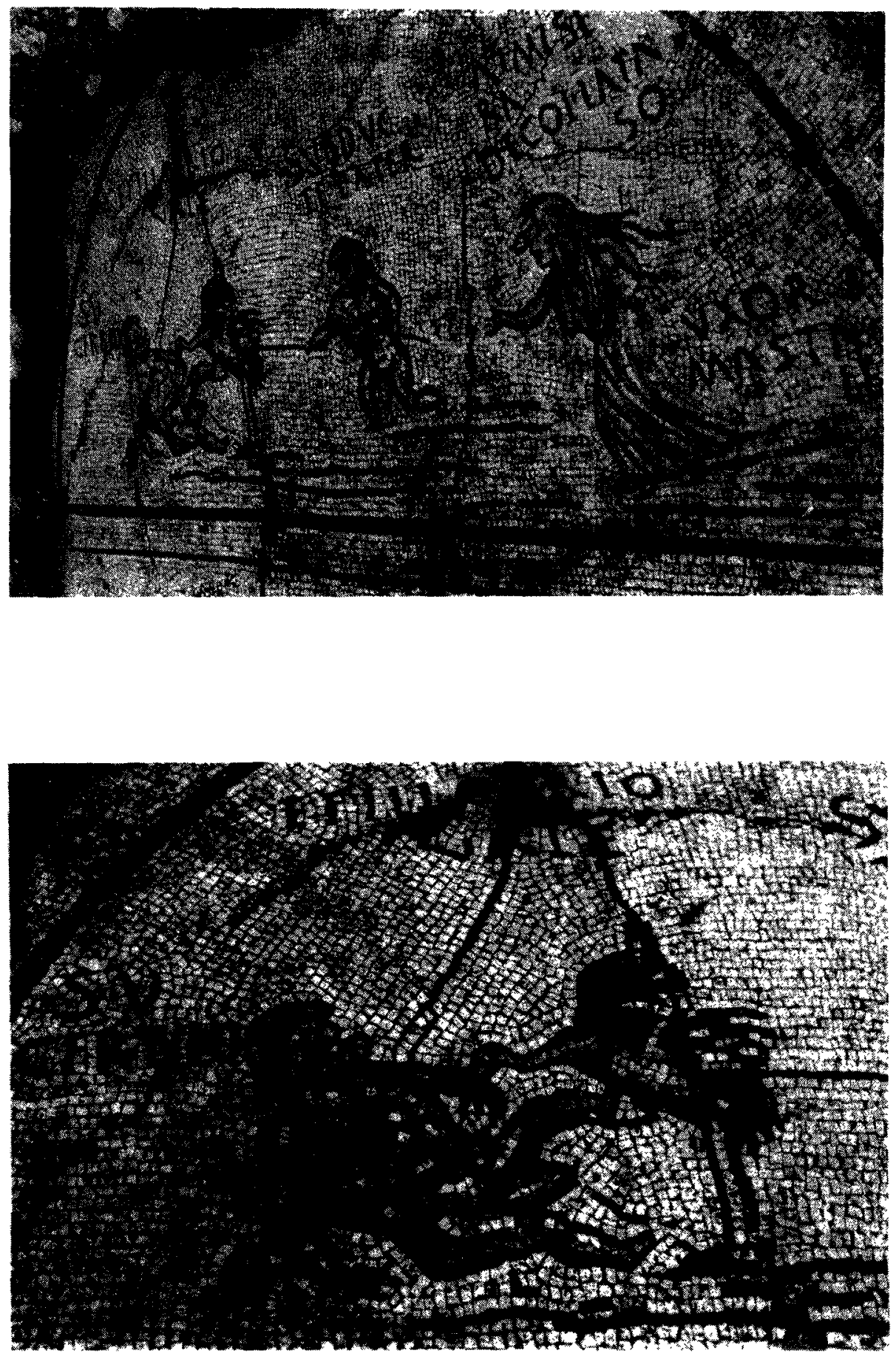

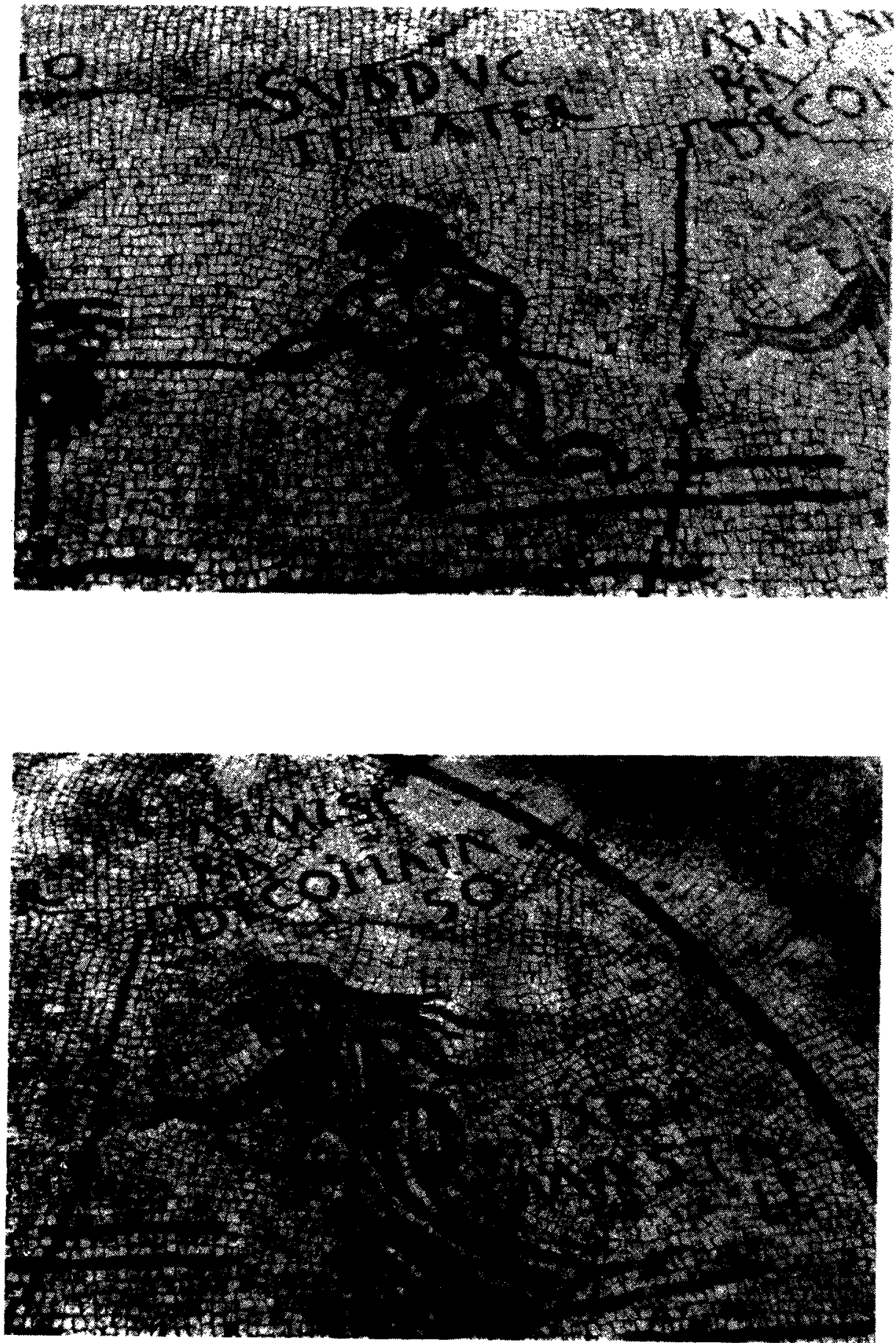
deux absides conservées avec ces répliques échangées par les personnages dans le feu de l'action constituent un hapax dans les représentations figurées grecques ou romaines en mosaïque comme en peinture.

On nous a demandé (1987) d'établir le texte et d'en faire la traduction commentée ${ }^{13}$. L'une des tâches qui incombe au traducteur est d'essayer d'appréhender et si possible de déterminer la référence littéraire de ces petites scènes.

Nous avons choisi de présenter ici la première séquence, celle du combat proprement dit entre les pygmées et leur ennemie héréditaire, scène qui illustre un échange de répliques entre un père pygmée (Cervius) aux prises avec l'oiseau et son fils (Géryon) accourant à son aide, en présence d'une femme (l'épouse Mastalé) qui exprime sa détresse. Soulignons au passage le caractère vulgaire de ce latin qui saute aux yeux avec l'omission du $-m$ et du $-s$ finals, la confusion de la semi-voyelle $-u-(=w)$ et du $-b-$, le changement du $-u$ - bref en $-o$ - bref plus ouvert, l'absence de l'aspiration initiale et enfin la transformation de l'upsilon en $i$. Voici maintenant une première traduction qui ne rendra cependant pas compte du sens de l'expression insolite decollata sum:

- Je suis Cervius. Ah! Géryon, mon fils, adieu!

- Tire-toi de dessous, père!

- Hélas, misère de moi, je suis decollata (l'épouse Mastalé). ${ }^{14}$

Observons-nous la représentation figurée d'une scène de comédie? Ainsi que nous l'avons rappelé, c'est la première fois que des inscriptions latines sont jointes à une illustration de géranomachie et cette insertion dans une série, dont la succession des tableaux s'apparente assez au mouvement scénique, donne naturellement plus de poids à l'hypothèse d'une interférence dramatique.

Et si nous avions devant les yeux comme le souvenir d'une pièce créée à l'époque même de la mosaïque, il ne pourrait s'agir que d'un extrait du mime (à ne pas confondre avec la pantomime dépourvue de texte), seul genre dramatique encore productif au III s. après J.-C. Mais il n'existe aucun indice sûr, ni littéraire ni figuré, de la présence des pygmées dans la distribution des acteurs du mime latin, ou encore de leur apparition, fût-elle rapide et

13. André Daviault, «Étude philologique et littéraire», Une mosaïque à inscriptions (Madrid, Publications de la Casa de Velázquez, 1987) pp. 55-78.

14. - Su Cerbio (=sum Ceruius). E fili Gerio (=Hem fili Geryo) uale! - Subduc te, pater! - Ai $(=E i)$ misera! decollata so $(=$ sum). Vxor Mastale. 
accessoire, dans un scénario de mime. Nous savons, il est vrai, qu'une pièce mimique s'intitulait Alexandrie, dont on n'a conservé que le titre évocateur à la fois de l'exotisme nilotique et de la capitale artistique d'où est venue la vogue des représentations figurées caricaturales des pygmées. On pourrait concevoir à la limite une utilisation rapide du thème par un metteur en scène désireux de tirer parti de son pouvoir évocateur, dans un genre qui ne répugnait pas aux allusions mythologiques. La chose est indémontrable et il faudrait dans ce cas expliquer pourquoi notre mosaiste n'aurait retenu de ce mime qu'un épisode somme toute secondaire.

L'examen de notre tableau ne révèle aucun trait exclusif au mime: on ne peut, par exemple, faire fond sur le petit nombre d'acteurs, car la plupart des scènes de Plaute ne requéraient que deux ou trois personnages; le dessin des pygmées nus sans masque et dotés d'un phallus démesuré correspond tout simplement à l'imagerie caricaturale des pygmées et à l'idée traditionnelle qu'on s'était formée de leur constitution; le nez proéminent de l'épouse de même que les grosses mamelles à l'origine de son nom sont caractéristiques de la matrone de Comédie et n'étaient nullement spécifiques des femmes du mime. La grande taille ${ }^{15}$ de cette femme l'apparente moins à une pygmée qu'à un personnage du théâtre régulier dont les longs cheveux défaits et la tunique à manches rappellent les acteurs tragiques: dans notre contexte cette tenue pourrait constituer un des éléments d'une parodie tragique au même titre que ses gestes désespérés et son gémissement de douleur. En somme l'uxor de Cervius ressemble moins à une femme du mime qu'à un personnage composite, qui emprunterait son visage repoussant ainsi que ses seins rebondis à la Comédie, alors que ses cheveux longs, son costume et son jeu pathétique seraient du ressort de la Tragédie.

Au cours de cette investigation aucune preuve solide n'est venue confirmer l'hypothèse d'une scène figurée du mime. Paradoxalement, au fur et à mesure que l'analyse disqualifiait le mime comme source unique d'inspiration, apparaissaient des signes, trop nombreux pour être fortuits, d'une influence générale du théâtre.

15. S'il est possible que la haute stature de Mastalé traduise de la part du mosaïste son souci de rester fidèle à la tradition aristotèlicienne de la supériorité en taille de la femelle sur le mâle chez les pygmées (Aristt., P.N. VI, 467a 32), la différence anatomique des époux nous semble révélatrice d'autres intentions: en plus d'avoir visé l'effet comique en accentuant l'incongruité d'un couple aussi mal assorti, l'artiste a délibérément dessiné un personnage de hauteur normale pour favoriser le recoupement souhaité avec les modèles de femmes du théâtre. 
Et l'empreinte du théâtre est encore plus sensible dans le texte, qui contribue à la uis comica de la représentation. Ainsi notre analyse va nous montrer l'importance du texte qui non seulement remplit la fonction d'auxiliaire de l'image, mais qui porte en lui-même sa propre «force comique»: le comique verbal est présent partout et d'abord dans les noms des personnages qui témoignent de l'intention satirique de l'artiste. Mastale procède d'un comique élémentaire: malgré son statut d'hapax cette dénomination n'a rien d'hermétique; transcription latine d'un mot grec féminin dérivé de mastós «mamelle», l'expression correspond au latin mammeata et signifie littéralement «qui a de grosses mamelles». Inutile d'ajouter que ce nom va bien à l'uxor, que l'artiste a délibérément figurée avec des seins proéminents. Le recours à un nom grec est typique de l'onomastique de la Comédie.

Le nom du fils Geryo possède une connotation espagnole importante et surtout une valeur ironique plus grande: on sait que Géryon en dehors de notre inscription n'apparaît que dans la mythologie. C'est le nom du géant au triple corps finalement tué par Hercule et qui habitait en Espagne sur l'île rouge d'Érythie située devant l'embouchure du Guadalquivir. Mais pourquoi avoir affublé un nabot du nom d'un géant légendaire? Tout simplement pour accentuer la petite taille du pygmée au moyen d'un nom antiphrastique, empruntant ainsi un procédé banal dans la Comédie latine.

Enfin la victime, le père, qui est le seul à porter un nom latin, se trouve appartenir à la gens Ceruia, qui chez les Romains avait la réputation de produire des représentants forts et terribles. La valeur comique d'un tel nom infligé à un bout d'homme incapable de vaincre un volatile est facile à saisir.

Pour ne laisser subsister aucun doute sur l'étroite parenté qui existe entre nos bandes dessinées et la Comédie latine, nous observerons que si, dans ce premier tableau le choix des expressions familières subduc te, «tire-toi de dessous», et ai misera "Hélas! misère de moi», évoque la langue des vieux comiques, un terme employé dans la deuxième abside, que nous n'avons pu montrer, nous y renvoie obligatoirement: c'est le mot erus, employé de préférence à dominus, pour désigner le maître. Ce mot est spécifique du théâtre et disparaît du latin au $\mathrm{I}^{e r} \mathrm{~s}$. avant J.-C.

Il est temps de terminer ce que nous avons commencé à propos du comique d'expression et d'essayer de faire un sort à l'énigmatique cri de désespoir decollata sum lancé par l'épouse et dont l'interprétation n'est pas évidente: on se demande pourquoi ce verbe remplace le tour habituel ai misera perii, «hélas malheureuse, je suis perdue»! 
Avant ou au lieu de céder à un réflexe bien naturel qui consisterait à rechercher derrière l'expression quelque jeu de mots ou double sens égrillard mais peut-être trop moderne, il convient d'abord d'enquêter sur le sens et les attestations de cette expression. Le verbe decollare n'est attesté qu'une fois à l'époque archaïque et dans un sens différent, "enlever du cou» ${ }^{16}$, qui n'est manifestement pas le nôtre ici. Notre acception decollare signifiant «décapiter» n'apparaît pas avant le début de l'Empire et, fait remarquable, elle n'est jamais prise au figuré comme ici: en effet, nous comprenons bien qu'en s'écriant quelle est décapitée, Mastalé veut dire qu'elle est perdue. Le hasard ne peut être invoqué pour expliquer le choix d'une expression si inusitée. Il est sûr que l'allusion au caput ou à la tête qu'elle prétend perdre cache un sens comique dans cette atmosphère de bouffonnerie et de parodie créée par le grotesque des figures ainsi que par le ridicule des noms: peut-être une plaisanterie résidant dans un jeu de mots sur le nom de Ceruius qui dans l'esprit de Mastalé serait tiré de ceruix, «cou, tête»? Mais il n'existe pas d'étymologie populaire de ce genre. À la réflexion l'équation Ceruius = caput n'est pas si extravagante dans le monde fantaisiste des pygmées. En effet ces petits êtres contrefaits étaient traditionnellement reproduits dans l'art grécoromain avec un corps ramassé qui laissait paraître leur tête disproportionnée par rapport à leur taille ${ }^{17}$. Quoique cette caractéristique ne soit pas aussi accusée dans nos mosaïques, il n'en demeure pas moins que la tête volumineuse était typique d'innombrables représentations figurées de pygmées. Et le fait que notre artiste n'ait pas exagéré ce détail dans l'exécution de son dessin n'empêche pas le texte de rendre mieux compte d'une constante morphologique connue de tout le monde.

Dans cette perspective nous croyons que la réplique de la matrone renferme également une référence bouffonne à la difformité céphalique du mari pygmée: Mastalé dit qu'elle ne perd pas un homme, mais un bout d'homme, une tête! Il s'y greffe peut-être un jeu de mots sur la décollation prise dans le sens de décapitation et la décollation entendue comme une séparation pour annoncer l'état de veuvage. De plus notre interprétation n'est pas exclusive d'une lecture plus libre favorisant d'autres associations ou allu-

16. Nonius, De compendiosa doctrina, 97 Lindsay: Decollare, de collo deponere: Caecilius, 116 Ribbeck habes, uide, tibi tradidi, in tuo collo est: decolles caue («tu l'as, regarde, je te l'ai passé, il est à ton cou, prends garde de l'en enlever».

17. Paul Monceaux, «la Légende des pygmées et les nains de l'Afrique équatoriale», Revue historique, XLVII, I, (Paris, 1891), p. 57; Pietro Janni, Etnografia e mito. La storia dei Pigmei (Rome, Edizioni dell'Ateneo e Bizarri, 1978), p. 44. 
sions: rien n'interdit de penser que notre inscription a pu être formulée de manière à suggérer un rapprochement facile avec le mot coleus, par exemple. De toute façon le traducteur n'a pas beaucoup de moyens pour rendre ce comique d'expression, si ce n'est celui de traduire platement par «je suis décapitée», quitte à renvoyer le lecteur à un commentaire infrapaginal.

C'étaient là quelques exemples significatifs du rapport particulier du traducteur au texte original latin et du type d'investigations sur lesquelles est fondée la compréhension du message.

Université Laval 